\title{
Efficient THz Generation by Nonlinear Interaction of Gaussian Laser Beam With the Anharmonic and Rippled CNTs Aligned Vertically in the Array
}

\section{Sandeep Kumar}

Lovely Professional University

Shivani Vij

DAV Institute of Engineering and Technology

Niti Kant

Lovely Professional University

Vishal Thakur ( $\square$ vishal20india@yahoo.co.in )

Lovely Professional University https://orcid.org/0000-0002-5347-6956

\section{Research Article}

Keywords: Rippled CNTs, THz radiation, static magnetic field, dielectric surface, Gaussian laser, nonlinear restoration force

Posted Date: November 10th, 2021

DOI: https://doi.org/10.21203/rs.3.rs-1061057/v1

License: (c) (i) This work is licensed under a Creative Commons Attribution 4.0 International License. Read Full License 


\title{
Efficient THz Generation by nonlinear interaction of Gaussian laser beam with the anharmonic and rippled CNTs aligned vertically in the array
}

\author{
Sandeep Kumar ${ }^{1}$, Shivani Vij ${ }^{2}$, Niti Kant ${ }^{1}$, and Vishal Thakur ${ }^{1 *}$ \\ 1. Department of Physics, Lovely Professional University, G.T. Road, Phagwara - 144411, \\ Punjab, India \\ 2. Department of Applied Sciences, DAV Institute of Engineering \& Technology, Jalandhar, \\ 144008, India
}

\section{Abstract}

We purpose a theoretical analysis for the generation of efficient terahertz $(\mathrm{THz})$ radiation by using the nonlinear interaction of Gaussian laser beam with vertically aligned anharmonic, and rippled carbon nanotubes (CNTs) array. This array of vertically aligned carbon nanotubes (VA-CNTs) is embedded on the base of the dielectric surface. The VA-CNTs have been magnetized by applying a static magnetic field mutually perpendicular to the direction of propagation of the Gaussian beam and length of CNTs. The Gaussian laser beam passing through the CNTs exerts a nonlinear ponderomotive force on the electrons of CNTs and provides them resonant nonlinear transverse velocity. This produces the nonlinear current which is further responsible for the generation of $\mathrm{THz}$ radiation. The anharmonicity plays a vital role in the efficient generation of $\mathrm{THz}$ radiation. The anharmonicity arises due to the nonlinear variation of restoration force on the various electrons of CNTs. This anharmonicity in the electrons of CNTs helps in broadening the resonance peak. We have observed that externally applied static magnetic field ( $110 \mathrm{kG}$ to $330 \mathrm{kG}$ ) also paves the way for the enhancement of the normalized THz amplitude. 
Keywords: Rippled CNTs, THz radiation, static magnetic field, dielectric surface, Gaussian laser, and nonlinear restoration force.

\section{E-mail:vishal20india@yahoo.co.in}

\section{Introduction}

One of the main tasks in the modern technical world is to develop compact and highly efficient terahertz generation sources. Such $\mathrm{THz}$ generation sources can bring revolutionary changes in many fields of science and technology, a few of these are THz-spectroscopy, security systems, medical and health sectors, etc. [1-5]. In the present century, researchers have provided variety of schemes for the efficient generation of $\mathrm{THz}$ radiations. In these schemes, $\mathrm{THz}$ radiations are generated using various methods, for example by employing laser coupling to anharmonic CNTs [6], by using a wiggler magnetic field on vertically aligned carbon nanotubes (VA-CNTs) [7], buy using anharmonic CNTs in the presence of static magnetic field [8], by using laser filaments in the presence of static electric and magnetic fields [9], etc. According to Batrakov et al. [10], THz radiations can be generated through CNTs by using a static electric field. Parashar and Sharma [11] have applied optical rectification in CNTs to generate THz radiations. The CNTs, when irradiated with laser beams provide one of the promising ways for the creation of compact and efficient sources of $\mathrm{THz}$ radiations. The extraordinary electrical conductivity, thermal conductivity, and compact dimensional characteristics of CNTs make them a front runner in the field of $\mathrm{THz}$ generation [12-16].

In the present paper, we develop an analytical model for the efficient $\mathrm{THz}$ generation by the interaction of Gaussian laser beam with the array of VA-CNTs in the presence of static magnetic field applied perpendicular to the direction of propagation of laser and length of CNTs. In $2^{\text {nd }}$ segment of the paper, we have derived the relation for the nonlinear current density, which is further responsible for the generation of $\mathrm{THz}$ radiation. In $3^{\text {rd }}$ segment of the paper, we have provided the $\mathrm{THz}$ wave dynamics to 
calculate the normalized THz electric field. The discussion of results and conclusion has been provided in the last segment of this paper.

\section{Evaluation of nonlinear current density}

Consider a vertical array of single-walled anharmonic CNTs nested in dielectric surface (glass) as shown in Fig. 1. To magnetize these CNTs, a static magnetic field is applied along the $y$-direction transverse to the direction of laser propagation (z-direction) and the longitudinal axis of CNTs (xdirection). The microwave plasma-enhanced chemical vapor deposition synthesizing (MPECVD) technique can be engineered to obtain the deformed VA-CNTs [17-20]. The CNTs obtained by the above mentioned technique are known as rippled VA-CNTs. These are very softer and more prone to ripples as compared to crystalline CNTs obtained by other available methods like the arc discharge technique [21-24]. In the above mentioned MPECVD technique, the dimensions and alignment of SWCNTs can be easily controlled [25, 26]. In this way, we can produce density ripples of desired period and size in the CNTs. The number of CNTs per unit area in the array is $\mathrm{n}_{\mathrm{q}}$ and corresponding modulated density of CNTs is $n_{q}=n_{0 q} e^{i q x}$, here the term $n_{0 q}$ is the amplitude and $q$ is the wavenumber of density ripples produced in CNTs. The free electron density of each CNT is $\mathrm{n}_{0}$. Each CNT is characterized by the inner radius $a$, outer radius $b$, and length $L$. Each SWCNT is normally shaped as a hollow cylinder of compact dimensions to determine the electrical conductivity [27]. As far as we are concerned with the response of SWCNTs to the transverse electric and magnetic fields of the laser beam, these nanotubes as solid cylindrical tubes [28, 29]. The amplitude modulated Gaussian laser beam of angular frequency $\omega$ and wavenumber $k$, having non-uniform intensity distribution propagates through VACNTs with electric field profile

$\vec{E}=\hat{x} E_{0}[1+\mu \cos \Omega(t-z / c)] e^{-i(\omega t-k z)}$, 
where $\mu$ is the modulation depth, $\Omega$ is the modulation frequency in the $\mathrm{THz}$ range, $\mathrm{c}$ is the speed of light and $\hat{x}$ is the unit vector. The intensity profile of the incident Gaussian laser beam is represented by the relation $\mathrm{E}_{0}^{2}=\mathrm{E}_{00}^{2} \mathrm{e}^{-\mathrm{x}^{2} / \mathrm{r}_{0}^{2}}$, here $\mathrm{r}_{0}$ is initial beam radius.

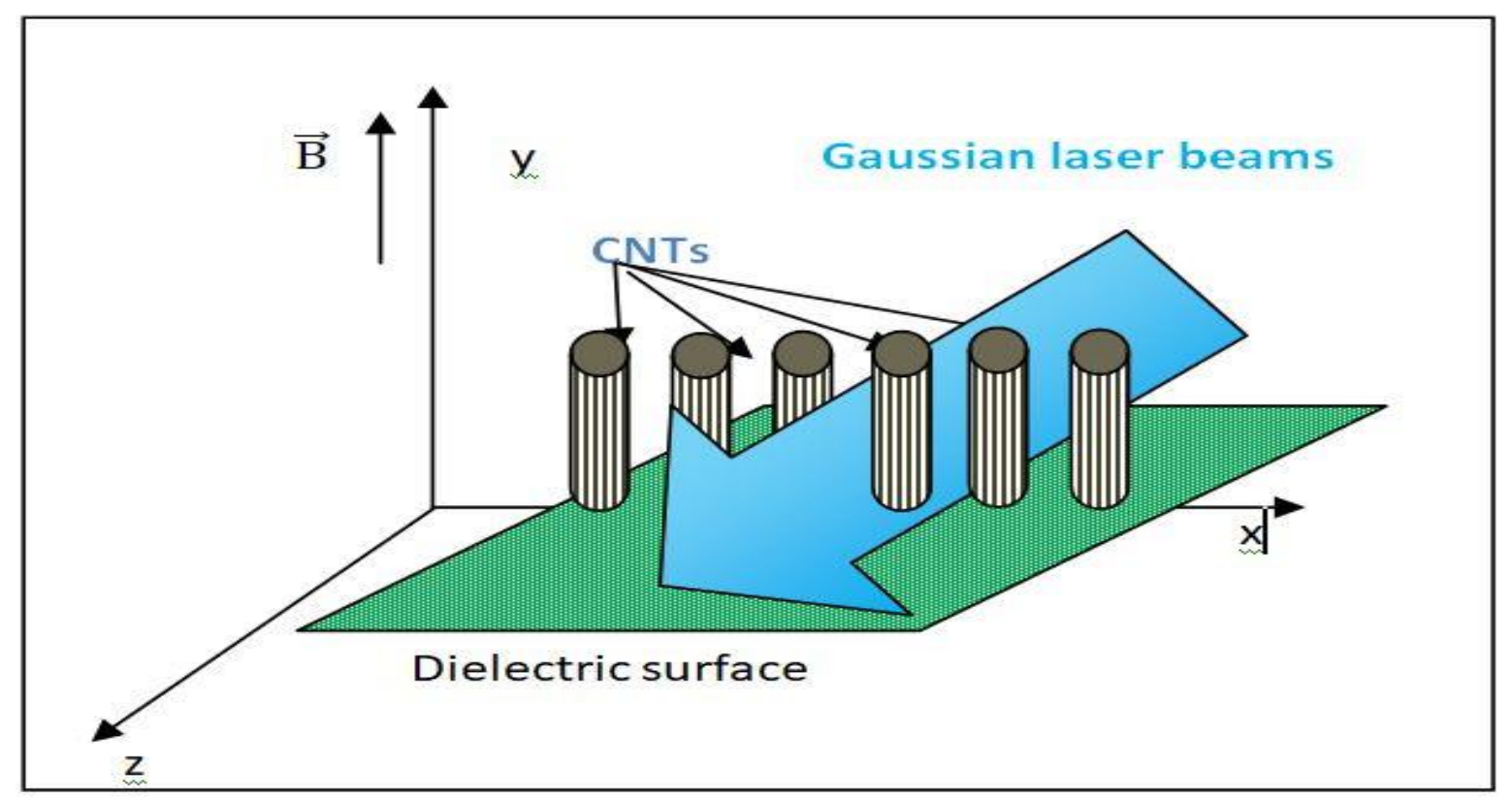

Fig. 1 Schematic representation of $\mathrm{THz}$ radiation generation from a rectangular array of horizontally aligned hollow CNTs nested in the glass plates under the effect of external magnetic and electric field

When the Gaussian laser beam interacts with the electrons of CNTs, provides oscillatory velocity to the electrons of CNTs. This oscillatory velocity of the electrons of CNTs is given as $\vec{v}=e \vec{E} / m i \omega$, here, e and $\mathrm{m}$ denote the electronic charge and mass respectively. The incident Gaussian beam also exerts a ponderomotive force on the electrons of CNTs as $\vec{F}_{\mathrm{PM}}=-\mathrm{e} \vec{\nabla} \phi_{\mathrm{PM}}$, where $\phi_{\mathrm{PM}}$ is the nonlinear ponderomotive potential. This nonlinear ponderomotive potential can be calculated by using the relation given $\phi_{\mathrm{PM}}=\left(-\mathrm{mvv}^{*} / 2 \mathrm{e}\right)$

$\phi_{\mathrm{PM}}=\frac{-\mathrm{e}}{4 \mathrm{~m} \omega^{2}} \mathrm{E}_{00}^{2}\left[1+2 \mu \cos \Omega\left(\mathrm{t}-\frac{\mathrm{z}}{\mathrm{c}}\right)\right] \mathrm{e}^{-\mathrm{x}^{2} / \mathrm{r}_{0}^{2}}$. 
By using the above equation (2), one can calculate $x$ and $z$ components of the nonlinear ponderomotive force in the exponential form. These components are acting perpendicular to the direction of the applied magnetic field (y-direction). These components are responsible for $\mathrm{THz}$ generation at resonance.

$$
\begin{aligned}
& \mathrm{F}_{\mathrm{x}}=\frac{\mu \mathrm{e}^{2} \mathrm{E}_{00}^{2} \mathrm{x}}{2 \mathrm{~m} \omega^{2} \mathrm{r}_{0}^{2}} \mathrm{e}^{-\mathrm{x}^{2} / \mathrm{r}_{0}^{2}} \mathrm{e}^{-i \Omega(\mathrm{t}-\mathrm{z} / \mathrm{c})} . \\
& \mathrm{F}_{\mathrm{z}}=\frac{-i \mu \mathrm{e}^{2} \mathrm{E}_{00}^{2} \Omega}{4 \mathrm{~m} \omega^{2} \mathrm{c}} \mathrm{e}^{-\mathrm{x}^{2} / \mathrm{r}_{0}^{2}} \mathrm{e}^{-i \Omega(\mathrm{t}-\mathrm{z} / \mathrm{c})} .
\end{aligned}
$$

The $\mathrm{x}$-component of the ponderomotive force $\mathrm{F}_{\mathrm{x}}$ is responsible for the oscillatory motion of the electrons of CNTs in a direction transverse to the direction of propagation of the laser, at the modulation frequency. This results in the shifting of the electron cylinder by the displacement $\vec{\Delta}$ from the ion cylinder along the x-direction as shown in Fig. 2. The overlapped region of the electron cylinder and the ion cylinder forms the space-charge field. The space charge field is not uniform. Hence, the restoration force experienced by the various electrons of the CNTs is not the same which further induces anharmonicity in the system of CNTs. Because of this CNTs become anharmonic. The spacecharge electric field produced by ion and electron cylinders is represented by $\overrightarrow{\mathrm{E}}_{+}$and $\overrightarrow{\mathrm{E}}_{-}$respectively. Thus the net electric field at the point $(r, \varphi, z)$ can be written as $\vec{E}=\vec{E}_{+}+\vec{E}_{-}$.

$$
\overrightarrow{\mathrm{E}}=\frac{\mathrm{n}_{0} \mathrm{e}\left(\mathrm{r}^{2}-\mathrm{a}^{2}\right)}{2 \epsilon} \frac{\overrightarrow{\mathrm{r}}}{\mathrm{r}^{2}}-\frac{\mathrm{n}_{0} \mathrm{e}}{2 \epsilon_{0}}\left[|\overrightarrow{\mathrm{r}}-\vec{\Delta}|^{2}-\mathrm{a}^{2}\right]\left[\frac{\overrightarrow{\mathrm{r}}-\vec{\Delta}}{|\overrightarrow{\mathrm{r}}-\vec{\Delta}|^{2}}\right]
$$

where, $\epsilon=\epsilon_{0} \epsilon_{\mathrm{r}}$ is known as the electric permittivity of the medium. As explained above, the $\mathrm{x}$ component of the ponderomotive force $\mathrm{F}_{\mathrm{x}}$ is responsible for the oscillatory motion of the electrons of CNTs along the $x$-direction, thus the expression for the corresponding $x$-component of the spacecharge electric field can be derived from Eq. (5). 


$$
\mathrm{E}_{\mathrm{X}}=\frac{\mathrm{n}_{0} \mathrm{e}}{2 \epsilon}\left[\left(1+\frac{\mathrm{a}^{2}}{\mathrm{r}^{2}}\right) \Delta_{\mathrm{x}}+\left(\frac{5 \cos \varphi}{\mathrm{r}}+\frac{4 \cos ^{2} \varphi}{\mathrm{r}}-\frac{\left(\mathrm{r}^{2}-\mathrm{a}^{2}\right)}{\mathrm{r}^{3}} \cos \varphi\right) \Delta_{\mathrm{x}}^{2}\right]
$$

The restoring force for the electrons of CNTs along can be obtained by using the relation, $\mathrm{F}_{\mathrm{Rx}}=-\mathrm{eE}_{\mathrm{x}}$,

$\mathrm{F}_{\mathrm{Rx}}=\frac{-\mathrm{n}_{0} \mathrm{e}^{2}}{2 \epsilon}\left[\left(1+\frac{\mathrm{a}^{2}}{\mathrm{r}^{2}}\right) \Delta_{\mathrm{x}}+\left(\frac{5 \cos \varphi}{\mathrm{r}}+\frac{4 \cos ^{2} \varphi}{\mathrm{r}}-\frac{\left(\mathrm{r}^{2}-\mathrm{a}^{2}\right)}{\mathrm{r}^{3}} \cos \varphi\right) \Delta_{\mathrm{x}}^{2}\right]$.

(7) This

restoration force is not the same for all the electrons of CNTs instead some of the electrons of CNTs experience a weak restoration force, whereas others experience a strong restoration force. This nonlinear behavior of restoration force is responsible for the anharmonicity in the CNTs. Hence, we need to calculate the $\varphi$ (average) and the $r$ (average) of restoration force to obtain it's linear $\left(\mathrm{F}_{\mathrm{LRx}}\right)$ and nonlinear components $\left(\mathrm{F}_{\mathrm{NLRx}}\right)$. These linear and nonlinear components of the restoration force are given as

$$
\left\langle\mathrm{F}_{\mathrm{LRx}}\right\rangle=-\frac{\mathrm{n}_{0} \mathrm{e}^{2}}{2 \epsilon} \frac{\int_{a}^{\mathrm{b}}\left(1+\frac{\mathrm{a}^{2}}{\mathrm{r}^{2}}\right) 2 \pi \mathrm{r} . \mathrm{dr}}{\int_{\mathrm{a}}^{\mathrm{b}} 2 \pi \mathrm{r} \cdot \mathrm{dr}} \Delta_{\mathrm{x}} \text { and }\left\langle\mathrm{F}_{\mathrm{NLRx}}\right\rangle=-\frac{\mathrm{n}_{0} \mathrm{e}^{2}}{2 \epsilon}\left(\frac{1}{2 \pi}\right) \int_{0}^{2 \pi}\left(\frac{5 \cos \varphi}{\mathrm{r}}+\frac{4 \cos ^{2} \varphi}{\mathrm{r}}-\frac{\left(\mathrm{r}^{2}-\mathrm{a}^{2}\right)}{\mathrm{r}^{3}} \cos \varphi\right) \mathrm{d} \varphi \Delta_{\mathrm{x}}^{2} .
$$

By using standard integrals, the above linear and nonlinear components can be simplified to get net average restoration force

$$
\left\langle\mathrm{F}_{\mathrm{LRx}}\right\rangle+\left\langle\mathrm{F}_{\mathrm{NLRx}}\right\rangle=\frac{-\mathrm{m} \omega_{\mathrm{P}}^{2}}{2 \epsilon_{\mathrm{r}}} \Delta_{\mathrm{x}}\left[1+\beta+\alpha \Delta_{\mathrm{x}}\right]
$$

where, $\omega_{\mathrm{P}}=\left[\mathrm{n}_{0} \mathrm{e}^{2} / \mathrm{m} \epsilon_{0}\right]^{1 / 2}$ is the plasma frequency. The terms $\alpha$ and $\beta$ are known as nonlinear anharmonic and characteristic parameters. The values of both parameters depend on the dimensions of CNTs. Both nonlinear parameters are represented by the relations $\beta=2 \log _{e}(b / a) /\left(b^{2} / a^{2}-1\right)$ and $\alpha=4 /(b+a)$. Terms $\alpha$ and $\beta$ are also responsible for nonlinear mixing in the response of the electrons of CNTs in the array. 
As the static magnetic field $\vec{B}$ is applied along the $y$-axis, therefore, magnetic force can be resolved into their $x$ and $z$ components, $F_{B x}=-e_{z} B / c$ and $F_{B z}=e v_{x} B / c$ respectively. Under the influence of electric fields of the lasers, an external static magnetic field $\overrightarrow{\mathrm{B}}$ and space charge electric field, the displacement of electrons in CNTs can be controlled by the following set of equations

$\frac{\mathrm{d}^{2} \Delta_{\mathrm{x}}}{\mathrm{dt}^{2}}+\frac{\omega_{\mathrm{P}}^{2}}{2 \epsilon_{\mathrm{r}}}\left(1+\beta+\alpha \Delta_{\mathrm{X}}\right) \Delta_{\mathrm{x}}+\frac{\mathrm{F}_{\mathrm{BX}}}{\mathrm{m}}+v \frac{\mathrm{d} \Delta_{\mathrm{x}}}{\mathrm{dt}}=\frac{-\mathrm{eE} \mathrm{E}_{\mathrm{x}}}{\mathrm{m}}$,

$\frac{\mathrm{d}^{2} \Delta_{\mathrm{z}}}{\mathrm{dt}^{2}}+\frac{\mathrm{F}_{\mathrm{Bz}}}{\mathrm{m}}+v \frac{\mathrm{d} \Delta_{\mathrm{z}}}{\mathrm{dt}}=\frac{-\mathrm{eE}_{\mathrm{z}}}{\mathrm{m}}$,

where, $v$ represents electron-neutral collision frequency, which is lesser than $\omega$.

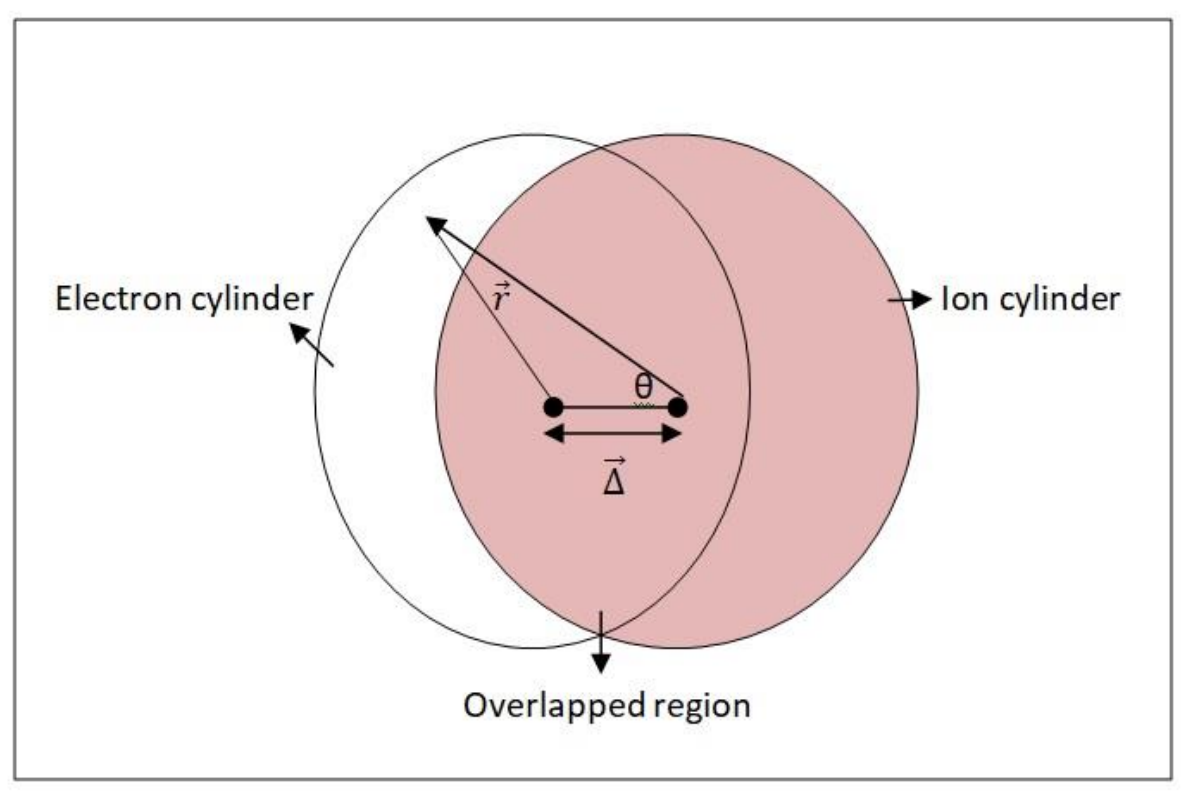

Fig. 2 shifting of the electron cylinder by displacement $\vec{\Delta}$ concerning the ion cylinder along the x-direction

On solving the equations (9) and (10), we obtain the $x$ and $z$ components for the displacement of the electrons of CNTs in the array

$\Delta_{\mathrm{x}}=\frac{-\left[(1+i v / \Omega) \mathrm{F}_{\mathrm{x}}+\left(i \omega_{\mathrm{c}} / \Omega\right) \mathrm{F}_{\mathrm{z}}\right]}{\mathrm{m}(1+i v / \Omega) \Omega^{2}\left[1-\omega_{\mathrm{P}}^{2}(1+\beta) / 2 \epsilon_{\mathrm{r}} \Omega^{2}-\omega_{\mathrm{c}}^{2} / \Omega^{2}(1+i v / \Omega)+i v / \Omega\right]}$, 
$\Delta_{\mathrm{z}}=\frac{-\left[\left(1-\omega_{\mathrm{P}}^{2}(1+\beta) / 2 \epsilon_{\mathrm{r}} \Omega^{2}+i v / \Omega\right) \mathrm{F}_{\mathrm{z}}-\left(i \omega_{\mathrm{c}} / \Omega\right) \mathrm{F}_{\mathrm{x}}\right]}{\mathrm{m}(1+i v / \Omega) \Omega^{2}\left[1-\omega_{\mathrm{P}}^{2}(1+\beta) / 2 \epsilon_{\mathrm{r}} \Omega^{2}-\omega_{\mathrm{c}}^{2} / \Omega^{2}(1+i v / \Omega)+i v / \Omega\right]}$,

where, $\omega_{c}=e B / m$ is known as cyclotron frequency of the electrons in CNTs.

With the help of the above equations (11) \& (12) and by using the fundamental relation of the velocity $\mathrm{v}=\mathrm{d} \Delta / \mathrm{dt}$ we can determine the corresponding nonlinear velocity components of the electrons of CNTs.

$\mathrm{v}_{\mathrm{X}}=\frac{i\left[(1+i v / \Omega) \mathrm{F}_{\mathrm{X}}+\left(i \omega_{\mathrm{c}} / \Omega\right) \mathrm{F}_{\mathrm{z}}\right]}{\mathrm{m}(1+i v / \Omega) \Omega\left[1-\omega_{\mathrm{P}}^{2}(1+\beta) / 2 \epsilon_{\mathrm{r}} \Omega^{2}-\omega_{\mathrm{c}}^{2} / \Omega^{2}(1+i v / \Omega)+i v / \Omega\right]}$

$\mathrm{v}_{\mathrm{z}}=\frac{i\left[\left(1-\omega_{\mathrm{P}}^{2}(1+\beta) / 2 \epsilon_{\mathrm{r}} \Omega^{2}+i v / \Omega\right) \mathrm{F}_{\mathrm{z}}-\left(i \omega_{\mathrm{c}} / \Omega\right) \mathrm{F}_{\mathrm{x}}\right]}{\mathrm{m}(1+i v / \Omega) \Omega\left[1-\omega_{\mathrm{P}}^{2}(1+\beta) / 2 \epsilon_{\mathrm{r}} \Omega^{2}-\omega_{\mathrm{c}}^{2} / \Omega^{2}(1+i v / \Omega)+i v / \Omega\right]}$.

One can use the relation $\mathrm{J}_{\omega}^{\mathrm{NL}}=-\mathrm{e} \mathrm{n}_{0} \mathrm{v}$ to calculate the nonlinear current density of the electrons of CNTs. The value of nonlinear current density is non-zero over the cross-sectional area of CNTs in the array. At the same time, its value is zero for the area lying in between the CNTs. Therefore we have calculated the average nonlinear current density of the array of CNTs by using the equation (3) \& (4) and the relation $J_{\mathrm{av} \cdot \omega}^{\mathrm{NL}}=-\mathrm{e} \mathrm{n}_{0} \mathrm{v}\left(\mathrm{n}_{\mathrm{q}}^{*} \pi\left(\mathrm{b}^{2}-\mathrm{a}^{2}\right)\right)$.

$\mathrm{J}_{\mathrm{av} . \Omega \mathrm{x}}^{\mathrm{NL}}=\frac{-\mathrm{in}_{0} \mathrm{n}_{0 \mathrm{q}} \pi\left(\mathrm{b}^{2}-\mathrm{a}^{2}\right) \mu \mathrm{e}^{3} \mathrm{E}_{00}^{2}\left[\mathrm{x}(1+i \mathrm{v} / \Omega) / \mathrm{r}_{0}^{2}+\Omega / 2 \mathrm{c}\right]}{2 \mathrm{~m}^{2} \Omega \omega^{2}(1+i v / \Omega)\left[1-\omega_{\mathrm{P}}^{2}(1+\beta) / 2 \epsilon_{\mathrm{r}} \Omega^{2}-\omega_{\mathrm{c}}^{2} / \Omega^{2}(1+i v / \Omega)+i v / \Omega\right]} \mathrm{e}^{-\mathrm{x}^{2} / \mathrm{r}_{0}^{2}} \mathrm{e}^{-i\left(\Omega \mathrm{t}-\mathrm{k}^{\prime} \mathrm{z}\right)}$

$\mathrm{J}_{\mathrm{av} . \Omega \mathrm{z}}^{\mathrm{NL}}=\frac{-\mathrm{n}_{0} \mathrm{n}_{0 \mathrm{q}} \pi\left(\mathrm{b}^{2}-\mathrm{a}^{2}\right) \mu \mathrm{e}^{3} \mathrm{E}_{00}^{2}\left[\Omega\left(1-\omega_{\mathrm{P}}^{2}(1+\beta) / 2 \epsilon_{\mathrm{r}} \Omega^{2}+i v / \Omega\right) /+\mathrm{x} \omega \mathrm{c} / \omega \mathrm{r}_{0}^{2}\right]}{2 \mathrm{~m}^{2} \Omega \omega^{2}(1+i v / \omega)\left[1-\omega_{\mathrm{P}}^{2}(1+\beta) / 2 \epsilon_{\mathrm{r}} \omega^{2}-\omega_{\mathrm{c}}^{2} / \omega^{2}(1+i v / \omega)+i v / \omega\right]} \mathrm{e}^{-\mathrm{x}^{2} / \mathrm{r}_{0}^{2}} \mathrm{e}^{-i\left(\Omega \mathrm{t}-\mathrm{k}^{\prime} \mathrm{z}\right)}$

The above nonlinear current density components represented by the equations (15) and (16) oscillates at frequency $\Omega$ and wavenumber $\mathrm{k}^{\prime}=\Omega / c-q$. This is different from the nonlinear ponderomotive force. However, with the ripples produced in CNTs have wave number q, there occurs matching of phase. 
Under this condition, resonant excitation of $\mathrm{THz}$ radiation can be realized. The nonlinear current density terms make a significant contribution to the $\mathrm{THz}$ field $\left(\mathrm{E}_{\mathrm{TH}}\right)$ and one can observe this contribution from the $\mathrm{THz}$ wave propagation equation.

\section{THz wave dynamics}

The wave equation is derived by using Maxwell's equations and describes the propagation of terahertz waves through the array of VA-CNTs.

$$
-\nabla^{2} \overrightarrow{\mathrm{E}}_{\mathrm{TH}}+\vec{\nabla}\left(\vec{\nabla} \cdot \overrightarrow{\mathrm{E}}_{\mathrm{TH}}\right)=\frac{-4 \pi \mathrm{i} \Omega}{\mathrm{c}^{2}} \vec{J}_{\mathrm{av} . \Omega}^{\mathrm{NL}}+\frac{\Omega^{2}}{\mathrm{c}^{2}} \vec{\epsilon}_{\mathrm{TH}} \cdot
$$

In the presence of an external static magnetic field applied along the y-axis in the collisional plasma of CNTs, the electric permittivity assumes the form of an anisotropic tensor.

$$
\vec{\epsilon}=\left|\begin{array}{ccc}
\epsilon_{\mathrm{xx}} & \epsilon_{\mathrm{xy}} & \epsilon_{\mathrm{xz}} \\
\epsilon_{\mathrm{yx}} & \epsilon_{\mathrm{yy}} & \epsilon_{\mathrm{yz}} \\
\epsilon_{\mathrm{zx}} & \epsilon_{\mathrm{zy}} & \epsilon_{\mathrm{zz}}
\end{array}\right| .
$$

The components of this anisotropic dielectric tensor have the following values

$\epsilon_{\mathrm{yz}}=\epsilon_{\mathrm{zy}}=\epsilon_{\mathrm{yx}}=\epsilon_{\mathrm{xy}}=0, \epsilon_{\mathrm{yy}}=1-\omega_{\mathrm{p}}^{2} / i \Omega(\mathrm{v}-i \Omega), \epsilon_{\mathrm{xx}}=\epsilon_{\mathrm{zz}}=1-\omega_{\mathrm{p}}^{2}(\mathrm{v}-i \Omega) / \mathrm{i} \Omega\left[(\mathrm{v}-i \Omega)^{2}+\omega_{\mathrm{c}}^{2}\right]$

and $\epsilon_{\mathrm{zx}}=-\epsilon_{\mathrm{xz}}=-i \omega_{\mathrm{c}} \omega_{\mathrm{p}}^{2} / \Omega\left[(v-i \Omega)^{2}+\omega_{\mathrm{c}}^{2}\right]$

With the use of the above components of dielectric tensor, Eq. (17) can be modified as:

$\frac{\Omega^{2}}{\mathrm{c}^{2}} \epsilon_{\mathrm{zz}} \mathrm{E}_{\mathrm{z}}-\frac{\Omega^{2}}{\mathrm{c}^{2}} \epsilon_{\mathrm{zX}} \mathrm{E}_{\mathrm{x}}=\frac{4 \pi i \Omega}{\mathrm{c}^{2}} \mathrm{~J}_{\mathrm{av} . \Omega \mathrm{z}}^{\mathrm{NL}}$

$-2 i \mathrm{k}^{\prime} \frac{\partial \mathrm{E}_{\mathrm{x}}}{\partial \mathrm{z}}+\left[\mathrm{k}^{\prime 2}-\frac{\Omega^{2}}{\mathrm{c}^{2}}\left(\epsilon_{\mathrm{xx}}+\frac{\epsilon_{\mathrm{zx}}^{2}}{\epsilon_{\mathrm{zz}}}\right)\right] \mathrm{E}_{\mathrm{x}}=\frac{4 \pi i \Omega}{\mathrm{c}^{2}} \mathrm{~J}_{\mathrm{av} \cdot \omega \mathrm{N}}^{\mathrm{NL}}+\frac{\epsilon_{\mathrm{zx}}}{\epsilon_{\mathrm{zz}}} J_{\mathrm{av} . \Omega \mathrm{z}}^{\mathrm{NL}}$ 
By using the phase-matching condition one can write

$$
\mathrm{k}^{\prime 2}-\frac{\Omega^{2}}{\mathrm{c}^{2}}\left(\epsilon_{\mathrm{xx}}+\frac{\epsilon_{\mathrm{Zx}}^{2}}{\epsilon_{\mathrm{zz}}}\right)=0 .
$$

With the help of the above equations (20) and (21) we can find the x-component of Thz electric field

$$
\mathrm{E}_{\mathrm{x}}=-\frac{-2 \pi \Omega}{\mathrm{k}^{\prime} \mathrm{c}^{2}}\left[\mathrm{~J}_{\mathrm{av} . \Omega \mathrm{x}}^{\mathrm{NL}}+\frac{\epsilon_{\mathrm{zx}}}{\epsilon_{\mathrm{zz}}} \mathrm{J}_{\mathrm{av} . \Omega \mathrm{z}}^{\mathrm{NL}}\right] \mathrm{x} .
$$

One can simplify the above equation of $\mathrm{THz}$ electric field by substituting the values of $\mathrm{J}_{\mathrm{av} \Omega . \mathrm{x}}^{\mathrm{NL}}$ and $\mathrm{J}_{\mathrm{av} . \Omega \mathrm{z}}^{\mathrm{NL}}$ from equations (15) and (16), to get normalized $\mathrm{THz}$ electric field expression

$$
\begin{aligned}
& \frac{\mathrm{eE}}{\mathrm{m} \omega \mathrm{c}}=\frac{\pi \mu}{\epsilon_{\mathrm{r}}}\left[\mathrm{n}_{\mathrm{q}} \pi\left(\mathrm{b}^{2}-\mathrm{a}^{2}\right)\right]\left(\frac{\Omega}{\mathrm{k}^{\prime} \mathrm{c}}\right)\left(\frac{\mathrm{n}_{0 \mathrm{q}}}{\mathrm{n}_{\mathrm{q}}}\right)\left(\frac{\mathrm{e} \mathrm{E}_{00}}{\mathrm{~m} \omega \mathrm{c}}\right)^{2}\left[(1+i v / \Omega)\left[1+\omega_{\mathrm{P}}^{2}(1+\beta) / 2 \epsilon_{\mathrm{r}} \Omega^{2}-\omega_{\mathrm{c}}^{2} / \Omega^{2}(1+i v / \Omega)+i v / \Omega\right]\right]^{-1}\left[\frac{i \mathrm{x}^{2}}{\mathrm{r}_{\mathrm{o}}^{2}}((1+\right. \\
& \left.\left.i \mathrm{v} / \Omega)-\frac{\left(\omega_{\mathrm{c}}^{2} / \Omega^{2}\right)\left(\omega_{\mathrm{p}}^{2} / \Omega^{2}\right)}{\left(\omega_{\mathrm{c}}^{2} / \Omega^{2}\right)-(1+i v / \Omega)^{2}+\left(\omega_{\mathrm{p}}^{2} / \Omega^{2}\right)(1+i v / \Omega)}\right)+\frac{i \mathrm{x} \omega_{\mathrm{c}}}{2 c}\left(1-\frac{\left(1+\omega_{\mathrm{p}}^{2}(1+\beta) / 2 \epsilon_{\mathrm{r}} \Omega^{2}+i v / \Omega\right) \omega_{\mathrm{p}}^{2} / \Omega^{2}}{\left(\omega_{\mathrm{c}}^{2} / \Omega^{2}\right)-(1+i v / \Omega)^{2}+\left(\omega_{\mathrm{p}}^{2} / \Omega^{2}\right)(1+i v / \Omega)}\right)\right] \mathrm{e}^{-\mathrm{x}^{2} / \mathrm{r}_{0}^{2}}
\end{aligned}
$$

\section{Results \& Discussion}

To perform numerical calculations, we have used a carbon dioxide laser beam with the following specified parameters. The angular frequency and wavelength of the laser beam is $\omega=1.78 \times$ $10^{14} \mathrm{rad} / \mathrm{s}$ and $\lambda=10.5 \mu \mathrm{m}$ respectively. The intensity of the laser beam is $\mathrm{I}=1.4 \times 10^{14} \mathrm{~W} / \mathrm{cm}^{2}$ with initial beam radius $r_{0}=40.0 \mu \mathrm{m}$. For this laser beam, the value of $\left(e E_{00} / m \omega c\right)$ is of the order of 0.06 . The length of each CNT in the array is $L=1.0 \mu \mathrm{m}$. In the array, each constituent CNT has an inner radius $\mathrm{a}=20 \mathrm{~nm}$ and outer radius $\mathrm{b}=40 \mathrm{~nm}$. The inter-tube separation in the array of VA-CNTs is $\mathrm{d}=$ $20 \mathrm{~nm}$. Corresponding to these specific dimensions of CNTs, the characteristic parameter $\beta$ has a value of 0.4631 . The relative permittivity of the glass substrate on which CNTs are grown is 3.5. The externally applied transverse static magnetic field lies in the range of $110 \mathrm{kG}$ to $330 \mathrm{kG}$. In figure 3 , we have plotted normalized $\mathrm{THz}$ field amplitude as a function of normalized $\mathrm{THz}$ frequency for modulation 
index $\mu=0.1$, the inner radius of CNT a $=20 \mathrm{~nm}$, the outer radius of CNT $\mathrm{b}=40 \mathrm{~nm}$, and inter-tube separation $\mathrm{d}=20 \mathrm{~nm}$ at various values of external static magnetic field $\mathrm{B}=110 \mathrm{kG}, 220 \mathrm{kG}$, and $330 \mathrm{kG}$. From this figure, it is evident that normalized THz amplitude increases with the increase of normalized $\mathrm{THz}$ frequency and reaches its peak value. If we still increase the normalized $\mathrm{THz}$ frequency, then normalized $\mathrm{THz}$ amplitude shows a decrease in its value from its peak value. The normalized $\mathrm{THz}$ amplitude attains its peak value at a particular normalized THz frequency, where surface plasmon resonance occurs. This resonance frequency condition is given by the relation $\omega=\omega_{\mathrm{p}}\left[(1+\beta) / 2 \epsilon_{\mathrm{r}}+\right.$ $\left.\omega_{\alpha}^{2}\right]^{1 / 2}$, where $\omega_{\alpha}=\omega_{c} / \omega_{p}$ is the ratio of cyclotron frequency to the plasma frequency of the electrons of CNTs. If one moves away from this resonance condition either on the left side or right side, then normalized THz amplitude shows a sharp decrease. This is because, at the resonance frequency, absorption of the laser beam by the CNTs becomes maximum. The external static magnetic field plays a key role in the enhancement of the normalized $\mathrm{THz}$ amplitude by increasing the nonlinearities in the array of VA-CNTs. Jain et al. [30] presented similar results, in the THz generation by the array of CNTs embedded on the metal surface under the effect of the external magnetic field. The surface plasmon resonance condition as mentioned above depends upon the value of the externally applied static magnetic field. This dependence shows that the surface plasmon resonance point slips towards the right of the normalized frequency with the increase of the static magnetic field. 


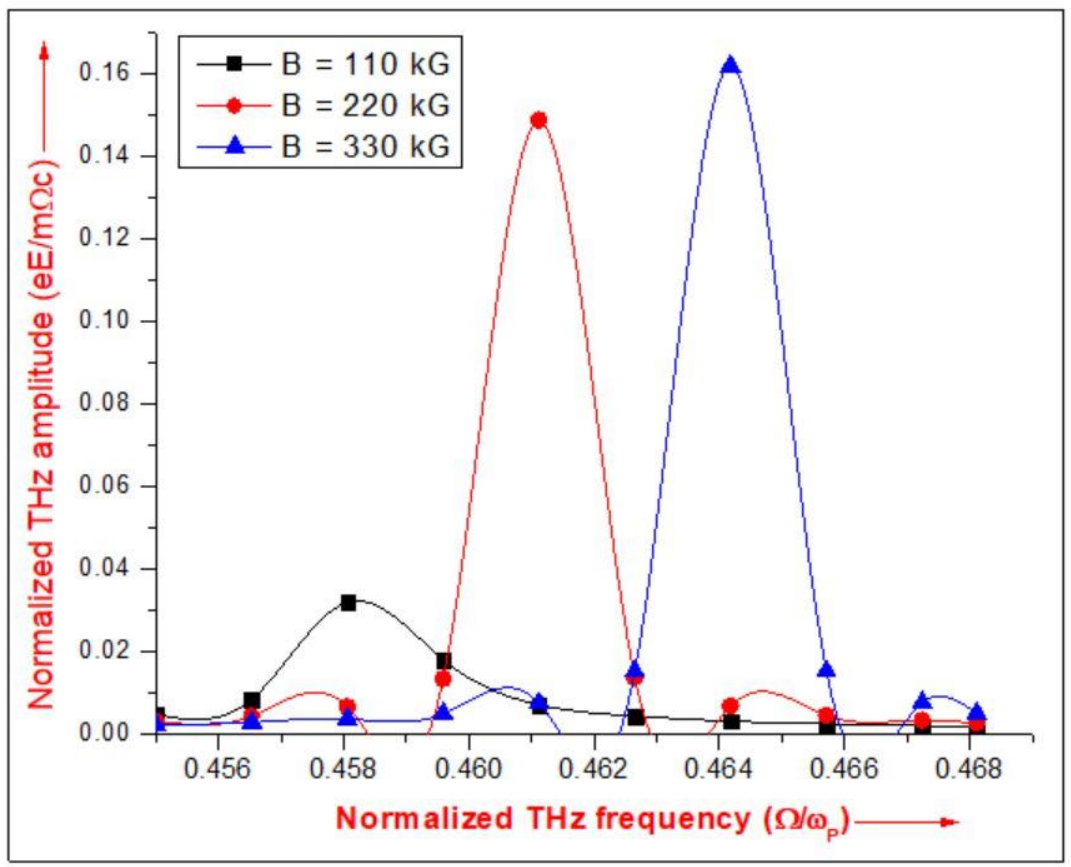

Fig. 3 Variation of normalized THz field amplitude with normalized THz frequency at various values of external static magnetic field $B=110 \mathrm{kG}, 220 \mathrm{kG}$ and $330 \mathrm{kG}$ for $\beta=0.4631, a=20 \mathrm{~nm}, \mathrm{~b}=40 \mathrm{~nm}$, and $\mu=0.1$.

To analyze the impact of dimensions of CNTs, we have plotted normalized THz field amplitude as a function of normalized THz frequency for modulation index $\mu=0.1$, the inner radius of CNT a $=20 \mathrm{~nm}$, the outer radius of CNT b $=40 \mathrm{~nm}, 30 \mathrm{~nm}, 25 \mathrm{~nm}$, and inter-tube separation $\mathrm{d}=20 \mathrm{~nm}$ at the optimized value of external static magnetic field $B=330 \mathrm{kG}$ as shown in figure 4 . Corresponding to the above values, we have three sets of $(a, b):(20 \mathrm{~nm}, 40 \mathrm{~nm}),(20 \mathrm{~nm}, 30 \mathrm{~nm})$, and $(20 \mathrm{~nm}, 25 \mathrm{~nm})$ with characteristic parameters $\beta=0.4631,0.6483$, and 0.7928 respectively. From the figure, it is evident that the normalized $\mathrm{THz}$ amplitude increases with the decrease in the value of characteristic parameter $\beta$. Out of three sets of $(\mathrm{a}, \mathrm{b})$, the THz amplitude is maximum for $(20 \mathrm{~nm}, 40 \mathrm{~nm})$ as compared to the other two sets. With the decrease in the value of the characteristic parameter, there occurs an increase in the size of CNTs. Larger size of CNTs means more absorption of the laser beam by the CNTs and hence results in the increase in the nonlinearities of the array of VA-CNTs. In the study of THz radiation by CNTs, Nemilentsau et al. [31] have explained the role played by external magnetic field and radii of 
CNTs for the efficient generation. Our results are showing the dependence of external magnetic field and radii of CNTs as presented in the figures $3 \& 4$. Our results are in consistent with the results presented by Nemilentsau et al. [31]. Watanabe et al. [32] have also shown $\mathrm{THz}$ electric field variation with radii of CNTs in their experimental work. From figure 4, it is clear that in each curve normalized $\mathrm{THz}$ amplitude has its peak value at the surface plasmon resonance point. Moreover, as the surface plasmon resonance condition depends upon the characteristic parameter $\beta$, therefore with the increase in the value of $\beta$, the surface plasmon resonance point slips towards the right side of the normalized $\mathrm{THz}$ frequency.

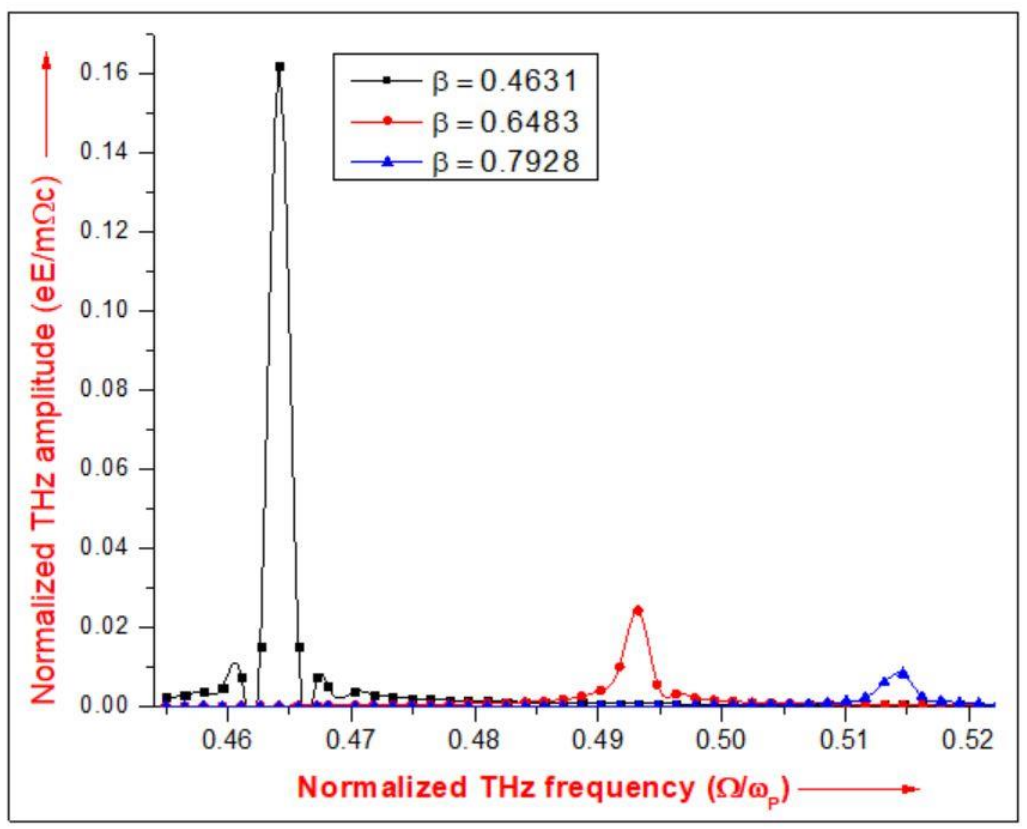

Fig. 4 Variation of normalized THz field amplitude with normalized THz frequency for different values of characteristic parameter $\beta$ at the optimized value of static magnetic field $B=330 \mathrm{kG}$.

In figure 5, we have shown a variation of normalized $\mathrm{THz}$ field amplitude with normalized $\mathrm{THz}$ frequency at various values of inter-tube separation $\mathrm{d}=20 \mathrm{~nm}, 25 \mathrm{~nm}$, and $30 \mathrm{~nm}$. The values of other parameters are kept the same as that of figure 3. From figure 5, one can observe a fall in the normalized $\mathrm{THz}$ amplitude with the increase in the inter-tube separation distance of CNTs in the array. The decrease in 
the inter-tube separation is responsible for the increase in the number density of CNTs in the array. This increase in the number density of CNTs results in the increase of the nonlinearities of the array of VA-CNTs. Vij et al. [7] has shown the similar results in their theoretical study of THz generation by using CNTs under the effect of a wiggler magnetic field. Also, the surface plasmon resonance condition $\omega=$ $\omega_{\mathrm{p}}\left[(1+\beta) / 2 \epsilon_{\mathrm{r}}+\omega_{\alpha}^{2}\right]^{1 / 2}$ is independent of the inter-tube separation distance $\mathrm{d}$, therefore in this graph surface plasmon resonance point remains same for the three curves.

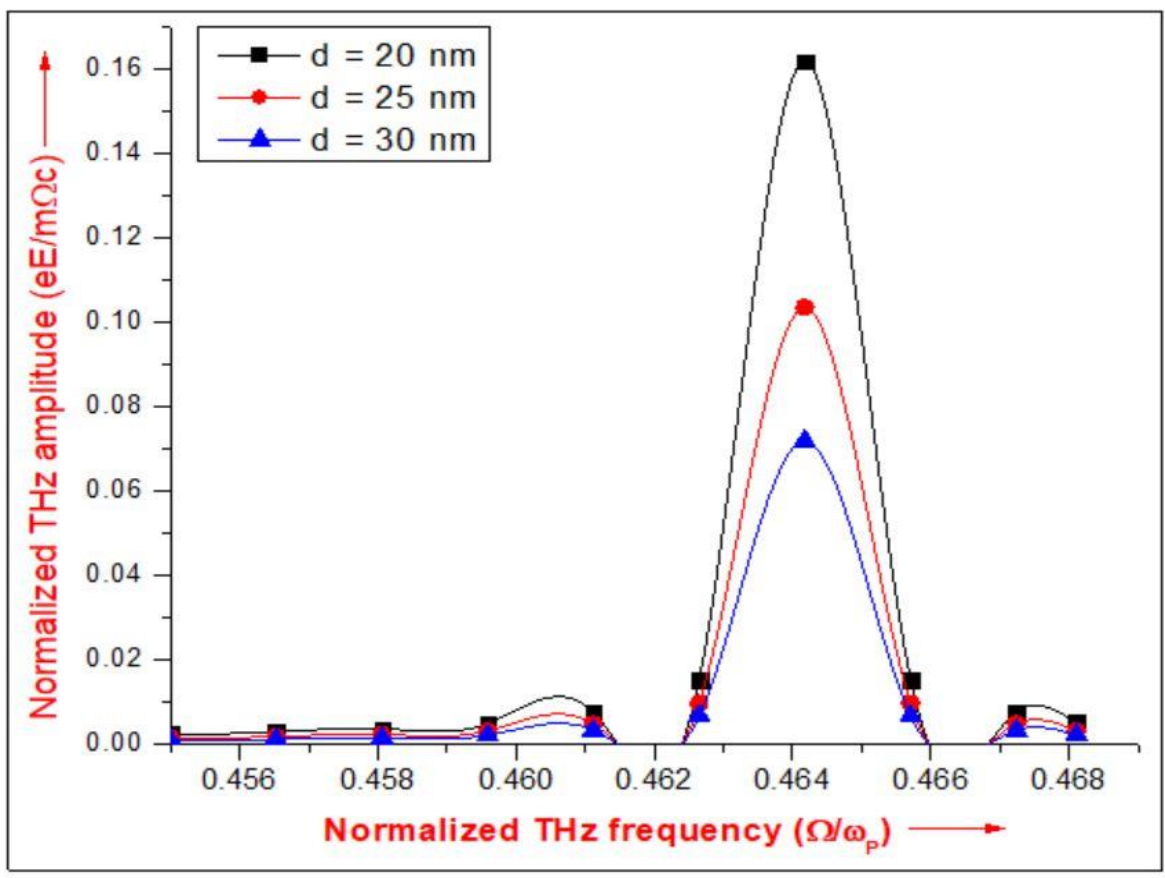

Fig. 5 Variation of normalized THz field amplitude with normalized THz frequency for different values of inter-tube separation at the optimized value of static magnetic field $B=330 \mathrm{kG}$ and characteristic parameter $\beta=0.4631$.

In figure 6, we have shown the variation of normalized $\mathrm{THz}$ amplitude with the normalized plasma frequency at different values of modulation indices of Gaussian laser beam $\mu=0.05,0.08$, and 0.1 and static magnetic field $\mathbf{B}=\mathbf{1 1 0} \mathbf{~ k G}, \mathbf{2 2 0} \mathbf{~ k G}, \mathbf{3 3 0} \mathbf{~ k G}$, where as the other parameters are kept same as 


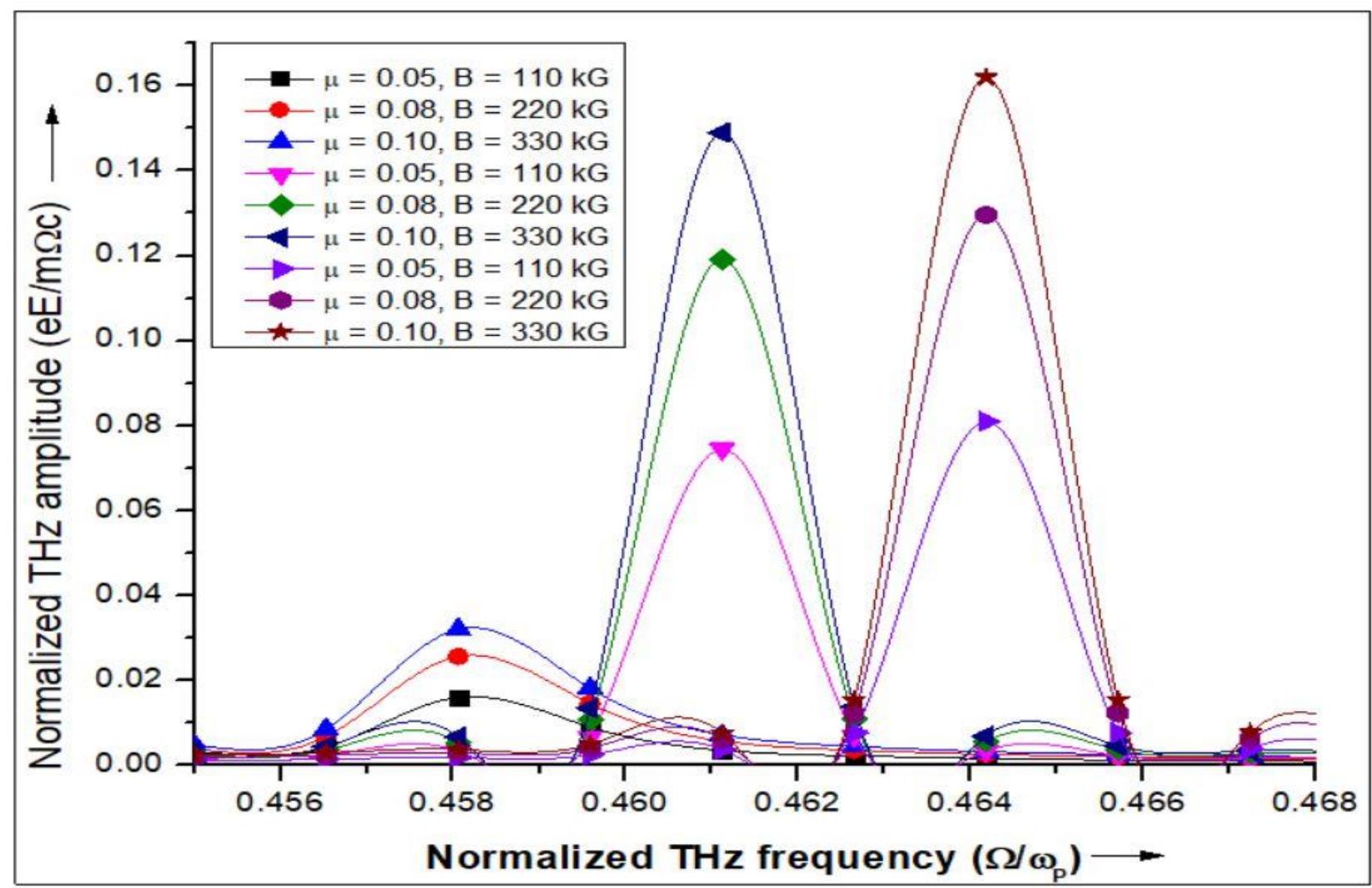

Fig. 6 Variation of normalized THz field amplitude with normalized plasma frequency for different values of modulation index and static magnetic field for the characteristic parameter $\beta=0.4631$.

that of figure 3. This graph shows that normalized $\mathrm{THz}$ amplitude increases with the increase of normalized plasma frequency and becomes maximum at the surface plasmon resonance point. For all the curves shown in figure 6 , the surface plasmon resonance point shifts towards a higher value. This is because of the increase in the value of the static magnetic field as explained above. Figure 6 , also explains the importance of modulation index, for the enhancement of normalized $\mathrm{THz}$ amplitude. Such dependence has also been explained by Kumar et al. [33] in their theoretical study THz generation by amplitude-modulated laser beam in ripple density plasma. So, the output of $\mathrm{THz}$ wave generation can be tuned by using appropriate values of the applied static magnetic field strength and modulation index. 


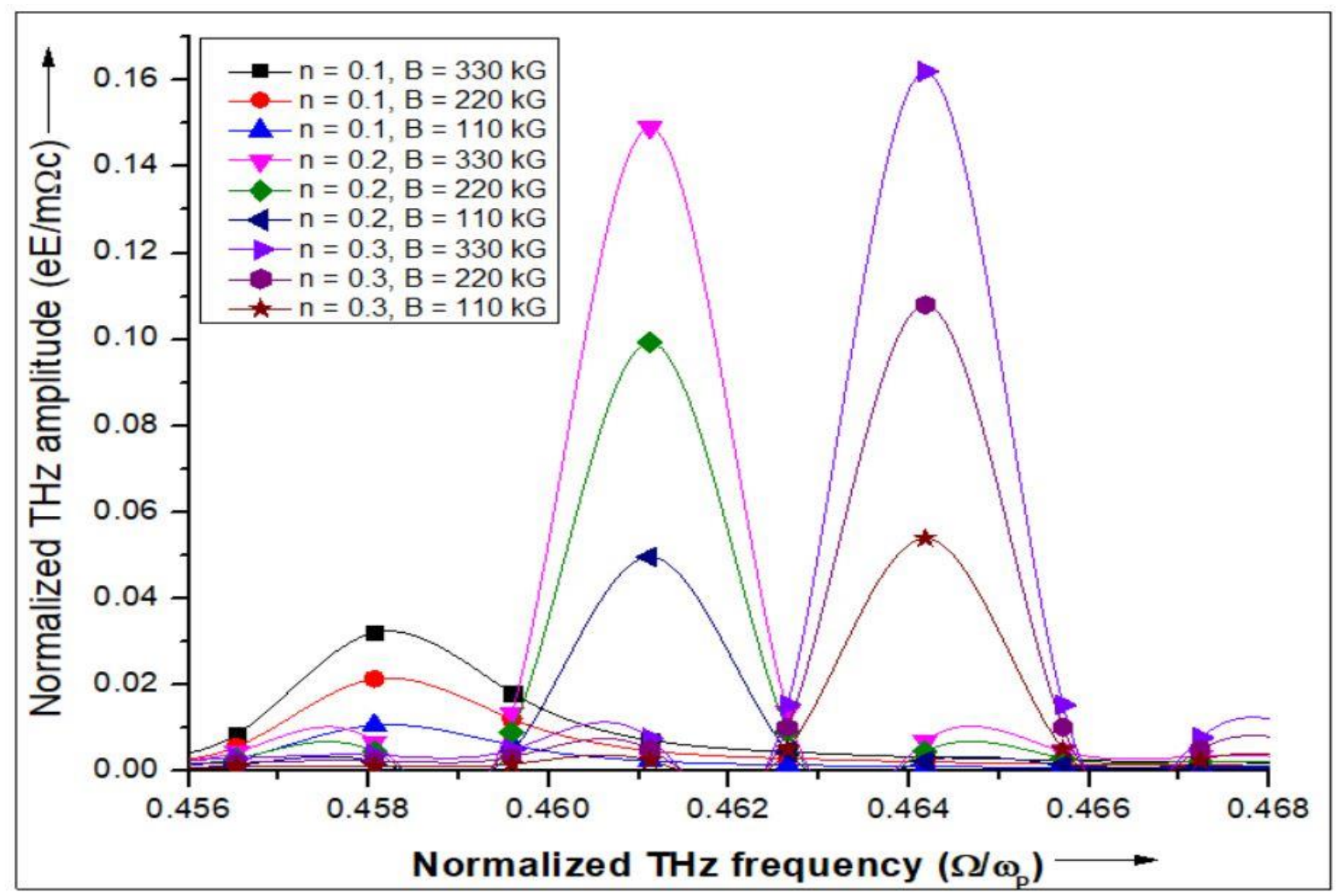

Fig. 7 Variation of normalized THz field amplitude with normalized THz frequency for different values of normalized ripple amplitude at the optimized value of the static magnetic field $B=330 \mathrm{kG}$ and the characteristic parameter $\beta=$ 0.4631.

To study the significance of density ripples, we plot the graph between normalized $\mathrm{THz}$ amplitude and normalized THz frequency for different values of normalized ripple amplitude $\left(n=n_{0 q} / n_{0}\right) \quad 0.1,0.2$, and 0.3 at the optimized value of the static magnetic field as shown in figure 7 . The values of other parameters are kept the same as that of figure 3. From the figure 7 , it is evident that there is a significant enhancement in the normalized $\mathrm{THz}$ amplitude with the increasing normalized ripple amplitude. The normalized $\mathrm{THz}$ amplitude becomes maximum at the optimized value of static magnetic field $\mathrm{B}=$ $330 \mathrm{kG}$ and normalized ripple amplitude $n=0.3$. The role of normalized ripple amplitude in enhancement of the normalized $\mathrm{THz}$ amplitude is quite reasonable as more and more electrons of 
CNTs become responsible for the generation of nonlinear current. A similar result has been observed by Malik et al. [34] in their theoretical work of generating the $\mathrm{THz}$ radiation by beating the Gaussian lasers in the presence of the static magnetic field.

Figure 8 shows the variation of normalized $\mathrm{THz}$ amplitude with normalized collision frequency for different values of static magnetic field $B=110 \mathrm{kG}, 220 \mathrm{kG}$, and $330 \mathrm{kG}$. The values of other parameters are kept the same as that of figure 3 . This graph unveils the adverse impact of normalized collision frequency on the normalized THz field amplitude. From this graph, it is evident that collisions between electrons and neutrals reduce the normalized $\mathrm{THz}$ amplitude. This is because the collisions between the electrons present in the CNTs result in the reduction of ponderomotive force and nonlinear current. However by applying a suitable magnetic field, one can overcome this loss up to some extent.

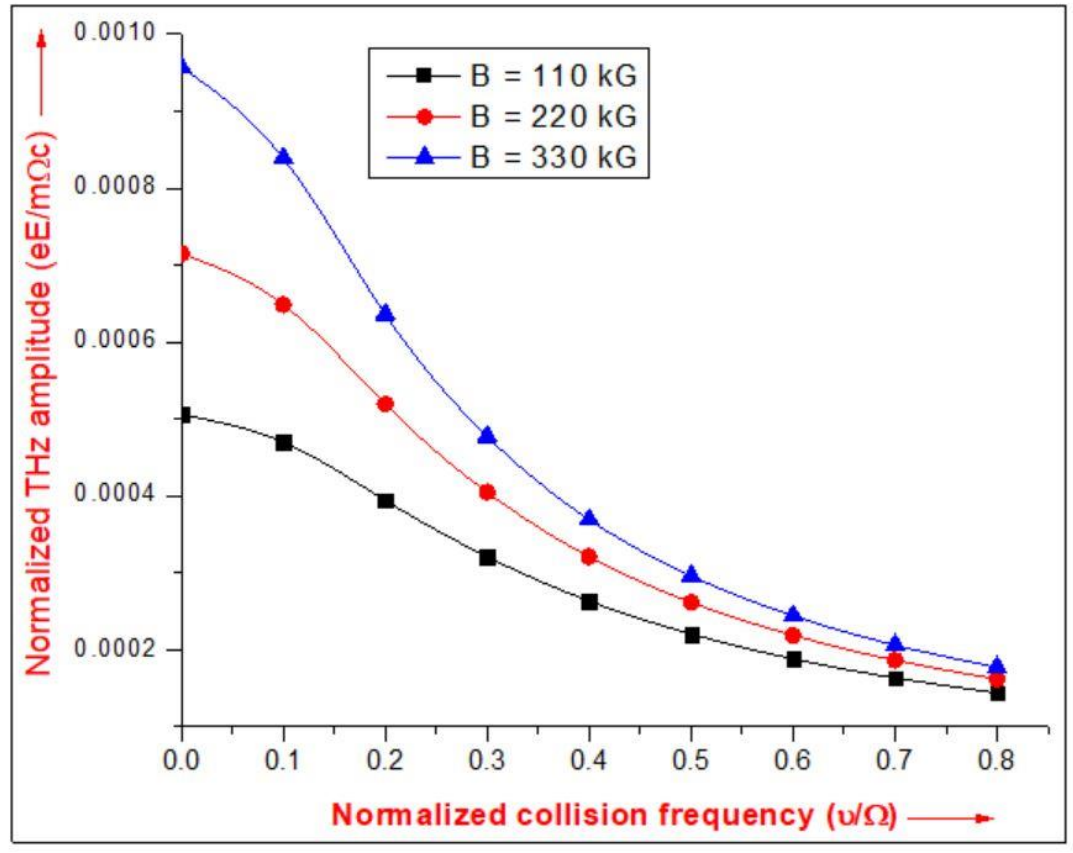

Fig. 8 Variation of normalized THz amplitude with normalized collision frequency for different optimized values of static magnetic field $B=110 \mathrm{kG}, 220 \mathrm{kG}$ and $330 \mathrm{kG}$ at characteristic parameter $\beta=0.4631$. 
Similar results have been shown by Singh and Malik [35] in their theoretical study of enhanced $\mathrm{THz}$ generation in magnetized collisional plasma.

\section{Conclusion}

The Gaussian laser beam makes a nonlinear interaction with VA-CNTs to resonantly excite THz radiation at the modulation frequency under the influence of an external magnetic field. This is responsible for the production of the nonlinear current which results in $\mathrm{THz}$ generation. The anharmonicity in the electrons of VA-CNTs helps in broadening the surface resonance peak. This also results in the further enhancement of $\mathrm{THz}$ generation. The surface plasmon resonance condition $\omega=$ $\omega_{\mathrm{p}}\left[(1+\beta) / 2 \epsilon_{\mathrm{r}}+\omega_{\alpha}^{2}\right]^{1 / 2}$ can be altered by varying the dimensions of CNTs and the strength of the applied static magnetic field.

\section{Author Contribution}

Sandeep Kumar: derivation, methodology and analytical modeling

Shivani Vij: graph plotting and writing

Niti Kant : numerical analysis and result discussion

Vishal Thakur: supervision, reviewing and editing

\section{Data Availability}

The data that supports the findings of this study are available insise the manuscript

\section{Declarations}

\section{Consent to participate}

Not applicable

\section{Consent for Publication}

Not applicable

\section{Conflict of Interest}




\section{References}

[1] C. Baker et al., "Detection of concealed explosives at a distance using terahertz technology," Proc. IEEE 95, 1559 (2007).

[2] A.R. Orlando and G.P. Gallerano, "Terahertz Radiation Effects and Biological Applications," J Infrared Millim Terahertz Waves 30, 1308 (2009).

[3] M.V Exter, C. Fattinger and D. Grischkowsky, "Terahertz time-domain spectroscopy of water vapor," Opt. Lett. 14, 1128-1130 (1989).

[4] B.H. Liu, Y. Chen, G.J. Bastiaans and X.C. Zhang, "Detection and identification of explosive RDX by THz diffuse reflection spectroscopy," Opt. Express 14, 415-423 (2006).

[5] Y.C. Shen, T. Lo, P.F. Taday, B.E. Cole, W.R. Tribe and M.C. Kemp, "Detection and identification of explosives using terahertz pulsed spectroscopic imaging," Appl. Phys. Lett. 86, 241116 (2005).

[6] S. Sharma S and A. Vijay, "Terahertz generation via laser coupling to anharmonic carbon nanotube array," Phys Plasmas 25, 023114 (2018).

[7] S. Vij, N. Kant and V. Thakur, "Resonant Enhancement of THz Radiation Through Vertically Aligned Carbon Nanotubes Array by Applying Wiggler Magnetic Field," Plasmonics 14, 1051 1056 (2019). 
[8] S. Kumar, S. Vij, N. Kant and V. Thakur, "Resonant Terahertz Generation by the Interaction of Laser Beams with Magnetized Anharmonic Carbon Nanotube," Plasmonics 2021 DOI $: 10.1007 / \mathrm{s} 11468-021-01529-z$

[9] S. Kumar, S. Vij, N. Kant, A. Mehta and V. Thakur, "Resonant terahertz generation from laser filaments in the presence of static electric field in magnetized collisional plasma," EPJ Plus 136, 148 (2021).

[10] K.G. Batrakov, O.V. Kibis, P.P. Kuzhir, M.R. da Costa,M.E. Portnoi, "Terahertz processes in carbon nanotubes," J. of Nanophotonics 4, 041665 (2014).

[11] J. Parashar and H. Sharma, "Optical rectification in a carbon nanotube array and terahertz radiation generation," Physica E. 44, 2069-2071 (2012).

[12] M.V. Shuba, G.Ya. Slepyan, S.A. Maksimenko, C. Thomsen and A. Lakhtakia, "Theory of multiwall carbon nanotubes as waveguides and antennas in the infrared and the visible regimes," Phys. Rev. B 79(15) 155403 (2009).

[13] G.W. Hanson, "Fundamental transmitting properties of carbon nanotube antennas," IEEE Trans. Antennas Propag. 53(11), 3426-3435 (2005).

[14] S.V. Bulyarskii, A.A. Dudin, A.P. Orlov, A.A. Pavlov and V.L. Leont'ev, "Forced vibration of a carbon nanotube with emission currents in an electromagnetic field," Tech. Phys. 62(11), 16271630 (2017).

[15] S.J. Hao. and G.W. Hanson, "Electromagnetic scattering from finite-length metallic carbon nanotubes in the lower IR bands," Phys. Rev. B 74(3), 035119 (2006). 
[16] M.E. Portnoi,O.V. Kibis, M.R. da Costa, "Terahertz applications of carbon nanotubes," Superlattices Microstruct. 43,399-407 (2008).

[17] H. Jackman, P. Krakhmalev and K. Svensson, "Mechanical behaviour of carbon nanotubes in the rippled and buckled phase" Journal of applied physics 117, 084318 (2015).

[18] I. Nikiforov et al., "Wavelike rippling in multiwalled carbon nanotubes under pure bending," Applied physics letters 96, 123107 (2010).

[19] C. G. Wang et al., "Buckling behaviour of carbon nanotubes under bending: From ripple to kink," Carbon 102, 224-235 (2016).

[20] S. Chang, T. Lin and C. Bai, "Low-temperature process in growing carbon nanotube," Microelectronics J. 38, 657-662 (2007).

[21] Z.F. Ren et al., "Synthesis of large arrays of well-aligned carbon nanotubes on glass," Science 1282, 1105 (1998).

[22] K. Y. Kim et al., "Coherent control of terahertz supercontinuum generation in ultrafast lasergas interactions" Nat. Photon 2, 605 (2008).

[23] H. K. Malik, "Density bunch formation by microwave in a plasma-filled cylindrical waveguide," Europhys. Lett. 106, 55002 (2014).

[24] C. C. Kuo et al., "Enhancement of Relativistic Harmonic Generation by an Optically Preformed Periodic Plasma Waveguide," Phys. Rev. Lett. 98, 033901 (2007).

[25] H. Dai, "Carbon nanotubes: opportunities and challenges," Surf. Sci. 500, 218-241 (2002).

[26] E. Muñoz, "Single-walled carbon nanotubes produced by cw CO2-laser ablation: study of parameters important for their formation," Appl. Phys. A 70, 145-151 (2000).

[27] S. Reich, C. Thomson, J. Maultzsch, "Carbon nanotubes: Basic Concepts and physical properties," Wiley VCH (2004).

[28] W. Lu, D. Wang, L. Chen, "Near-static dielectric polarization of individual carbon nanotubes," Nano Lett. 7, 2729 (2007). 
[29] C. S. Liu and V. K. Tripathi, "Observational consequences of parametrically driven vibrations of carbon nanotubes," Phys. Rev. B 70,115414 (2004).

[30] S. Jain, J. Parashar and R. Kurchania, "Effect of magnetic field on terahertz generation via laser interaction with a carbon nanotube array" Int. Nano Lett. 35326 (2013).

[31] A. M. Nemilentsau, G. Y. Slepyan, S. A. Maksimenko, O. V. Kibis, M. E. Portnoi, “Handbook of nanophysics volume 4 Nanotube and nanovires edited by K.D. Sattler CRC Press Newyork" Chapter 5, 5.1-5.15 (2010).

[32] S. Watanabe, N. Minami and R. Shimano Opt. Express 191528 (2011).

[33] S. Kumar, R. K Singh, M. Singh and R. P. Sharma, "THz radiation by amplitude-modulated self-focused Gaussian laser beam in ripple density plasma" Laser and Particle Beams 7 1-7 (2015).

[34] A. K Malik, H. K Malik and U. Stroth, "Terahertz radiation generation by beating of two spatialGaussian lasers in the presence of a static magnetic field" Physical Review E 85016401 (2012).

[35] D. Singh and H. K. Malik, "Enhancement of terahertz emission in magnetized collisional plasma", Plasma Sources Sci. Technol. 24045001 (2015). 